\title{
A KALMAN-TRACKER-BASED BAYESIAN DETECTOR FOR RADAR INTERFERENCE IN RADIO ASTRONOMY
}

\author{
Weizhen Dong, Brian D. Jeffs \\ Brigham Young University \\ Provo, UT 84606 \\ wzhdong@hotmail.com, bjeffs@ee.byu.edu
}

\author{
J. Richard Fisher \\ National Radio Astronomy Observatory \\ P.O. Box 2, Green Bank, WV 24944 \\ rfisher@nrao.edu
}

\begin{abstract}
Radio astronomical observations of important L-band spectral lines must often be made at frequencies allocated to pulsed air surveillance RADAR in the $1215-1350 \mathrm{MHz}$ band. Such pulsed interference must be dealt with at the Green Bank Telescope (GBT) and other observatories by "blanking" to remove corrupted data. This paper presents a new algorithm which improves aircraft echo detection by using a Kalman tracker to follow the path of a sequence of echoes. This Bayesian method enables more sensitive weak echo detection. Track information is used to form a spatial prior probability distribution for the presence of echoes in the next antenna sweep. A lower detection threshold is used in higher probability regions to pull out low level pulses without increasing the overall probability of false alarm detection.
\end{abstract}

\section{INTRODUCTION}

Air surveillance RADAR transmissions such as those from the ARSR-3 system occur at frequencies of importance for radio astronomy. Red shifted Hydrogen line observations range in frequency from 960 to $1400 \mathrm{MHz}$. These signals may dramatically disturb radio astronomical observations, and have been reported to be a significant problem at the Green Bank Telescope (GBT) [1], Arecibo [2] [3], and other observatories. However, the induced pollution is impulsive and transient, so for radio astronomy observation, one solution is to "time-blank" by simply not including RADARpulse-corrupted data samples during spectrum estimation [4].

Though echoes from fixed terrain objects (like local mountains) are easily anticipated and removed from observation data, aircraft echoes are not stationary and must be detected before they can be blanked. Aircraft hundreds of kilometers from the telescope cause troublesome echoes. A window of data surrounding each detected aircraft echo is removed,

This work is supported by the National Science Foundation grant number AST-9987339

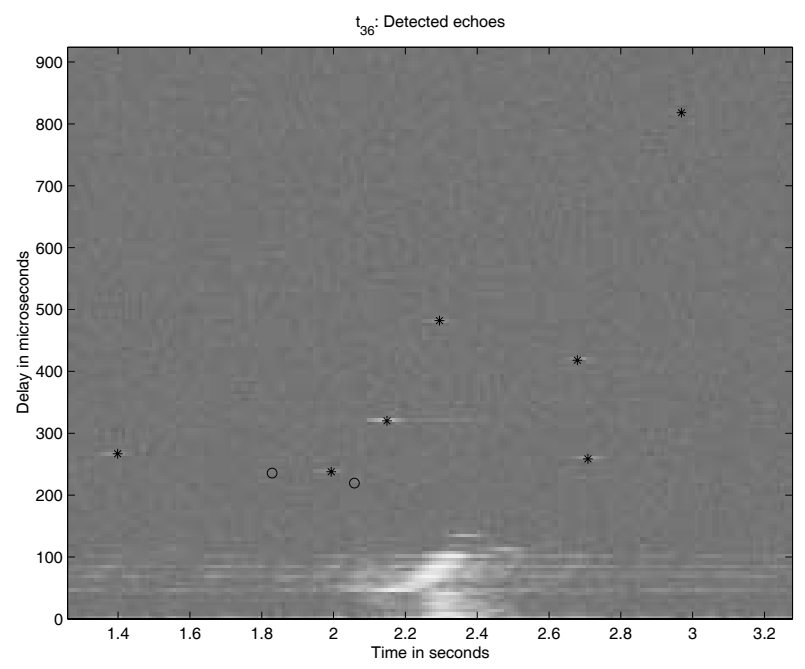

Fig. 1. Typical RADAR sweep snapshot frame as seen at the GBT. Aircraft echoes detected using a constant threshold $\tau_{0}$ are marked by stars. Two additional weak echoes found with the Bayesian detector are marked with circles.

including transmit antenna beampattern sidelobes. Because astronomers observe deep space signals which can be 40 $\mathrm{dB}$ or more below the noise floor, impulsive aircraft echoes weak enough to make detection difficult may still cause significant corruption to the data set. Thus high performance detection is a must.

Figure 1 shows $1292 \mathrm{MHz}$ band time series data collected at the GBT which has been reordered into rangebearing bins by synchronizing with the transmitter pulse repetition and antenna sweep rates. Both local terrain clutter and aircraft echoes are seen.

This paper presents a new Bayesian method for pulse detection based on Kalman filter tracking of aircraft echo motion. Time-history information across multiple past RADAR antenna sweeps is exploited to predict the location of the next echo. This prediction is used to form a prior probability distribution for pulse arrivals, which in a Bayesian 
framework improves weak echo detection.

\section{KALMAN TRACKER OVERVIEW}

A classical Kalman filter approach as often used for RADAR target following was implemented with some modifications [5]. The full Kalman tracker design is described in [6] [7]. For this discussion we need know only the basic Kalman prediction equations because they are used to form the echo prior probability distribution.

The true state vector at the $n^{\text {th }}$ "snapshot" (corresponding to an overhead pass of the transmit antenna beampattern at $\left.t_{n}\right)$ is defined as $\boldsymbol{x}(n)=[x(n) y(n) \dot{x}(n) \dot{y}(n)]^{T}$, where $x$ and $y$ are a single aircraft's position coordinates with velocities $\dot{x}$ and $\dot{y}$. The assumed aircraft dynamical model is $\boldsymbol{x}(n+1)=\boldsymbol{F} \boldsymbol{x}(n)+\boldsymbol{G} \boldsymbol{a}(n)$, where $\boldsymbol{F}$ is the state transition matrix, $\boldsymbol{G}$ is the process transfer matrix, and $\boldsymbol{a}(n)$ is the accelleration vector which models non-linear maneuvers. For each track, a new observation snapshot initiates an iteration of the Kalman filter which produces an updated state vector estimate, $\hat{\boldsymbol{x}}(n \mid n)$, and state error covariance matrix $\boldsymbol{P}(n \mid n)$. Then the predicted state at $t_{n+1}, \hat{\boldsymbol{x}}(n+1 \mid n)$, for the next (as yet undetected) echo, is computed as

$$
\begin{aligned}
\hat{\boldsymbol{x}}(n+1 \mid n) & =\boldsymbol{F} \hat{\boldsymbol{x}}(n \mid n), \text { and } \\
\boldsymbol{P}(n+1 \mid n) & =\boldsymbol{F} \boldsymbol{P}(n \mid n) \boldsymbol{F}^{T}+\boldsymbol{G} \boldsymbol{Q} \boldsymbol{G}^{T}
\end{aligned}
$$

where $Q$ is the process (acceleration) covariance. We note that the state vector is represented in Cartesian coordinates, while the observation is in polar range and bearing. For this reason an extended Kalman filter implementation is used which linearizes about the observation at each snapshot.

The desired tracker outputs are a prediction point where the next detection is expected $\left(\hat{x}_{n+1 \mid n}, \hat{y}_{n+1 \mid n}\right)$ which is just the first two elements of $\hat{\boldsymbol{x}}(n+1 \mid n)$, and shape parameters for an elliptical uncertainty region, $\mathcal{S}$, centered on this point (see Figure 2). The size of $\mathcal{S}$ depends on the quality of the track, and gets larger with an increase in observation noise, missed snapshot detections, or acceleration of the target. $\mathcal{S}$ selects the region of increased prior probability for an arriving echo pulse in snapshot $n+1$. $\mathcal{S}$ has radii $r_{x}$ and $r_{y}$ proportional to prediction error standard deviations $\sqrt{\boldsymbol{P}_{1,1}(n+1 \mid n)}$ and $\sqrt{\boldsymbol{P}_{2,2}(n+1 \mid n)}$ respectively.

Figure 2 illustrates this behavior. The plot shows track evolution for real GBT data over eight snapshots with multiple, overlapping aircraft tracks. The ellipses show prediction regions, $\mathcal{S}$ for each established track. Note the variety of sizes, corresponding to variations in track quality.

\section{PULSE ARRIVAL PRIOR DISTRIBUTION}

We assume that for each existing track, exactly one echo will occur (with probability one) at $t_{n+1}$, somewhere in the

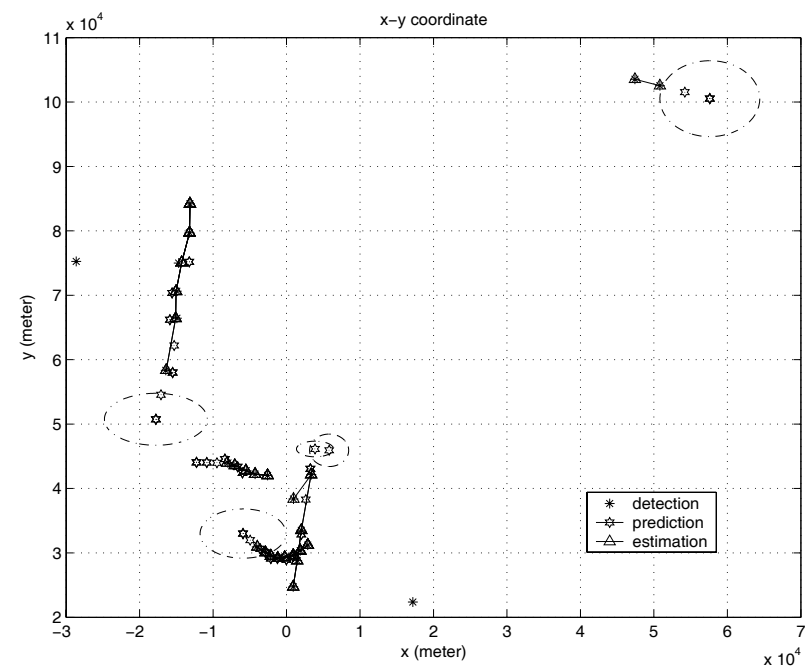

Fig. 2. Typical Kalman tracking performance for GBT data. Tracks have been automatically established and plotted for five aircraft. Prediction regions for the next snapshot, $\mathcal{S}$, are shown by dashed ellipses centered on prediction points, $\left(\hat{x}_{n+1 \mid n}, \hat{y}_{n+1 \mid n}\right)$. One track has been dropped due to lack of detections, and has no prediction point.

field, $\Omega$, of all range-bearing bins of interest. Let $E$ be the event that this echo occurs in partition $A \in \Omega$. We define a spatial probability density function, $f_{E}(x, y)$, such that $P(E)=\iint_{A} f_{E}(x, y) d x d y$. The following truncated twodimensional Gaussian distribution is proposed,

$$
\begin{aligned}
& f_{E}(x, y)= \\
& \begin{cases}\frac{1}{2 \pi \beta r_{x} r_{y}} e^{-\left[\frac{\left(x-\hat{x}_{n+1 \mid n}\right)^{2}}{2 \beta r_{x}^{2}}+\frac{\left(y-\hat{y}_{n+1 \mid n}\right)^{2}}{2 \beta r_{y}^{2}}\right]}, & (x, y) \in \mathcal{S}, \\
\frac{1-P_{\mathcal{S}}}{|\Omega|-|\mathcal{S}|}, & (x, y) \in \overline{\mathcal{S}}\end{cases}
\end{aligned}
$$

where

$$
\begin{aligned}
\mathcal{S} & =\left\{(x, y) \mid \frac{\left(x-\hat{x}_{n+1 \mid n}\right)^{2}}{r_{x}^{2}}+\frac{\left(y-\hat{y}_{n+1 \mid n}\right)^{2}}{r_{y}^{2}} \leq 1\right\}, \\
\Omega & =\mathcal{S} \cup \overline{\mathcal{S}} \text { (i.e. entire range-bearing map), } \\
P_{s} & =\iint_{\mathcal{S}} f_{E}(x, y) d x d y, \text { and }|\mathcal{S}|=\iint_{\mathcal{S}} d x d y .
\end{aligned}
$$

$\beta$ is set by design to control the height of the probability discontinuity at the boundary of $\mathcal{S}$. This model is illustrated in Figure 3.

The shape of $f_{E}(x, y)$ was selected as an arbitrary design choice, but it possesses the following desirable characteristics: 1) probability decreases with distance from the prediction point, 2) the distribution is smooth over $\mathcal{S}, 3$ ) density surface shape depends on prediction error covariance, $\boldsymbol{P}_{n+1 \mid n}$, so higher prediction error leads to lower probability density, and 4) there is a low, uniform probability of echoes arriving outside the prediction region. 


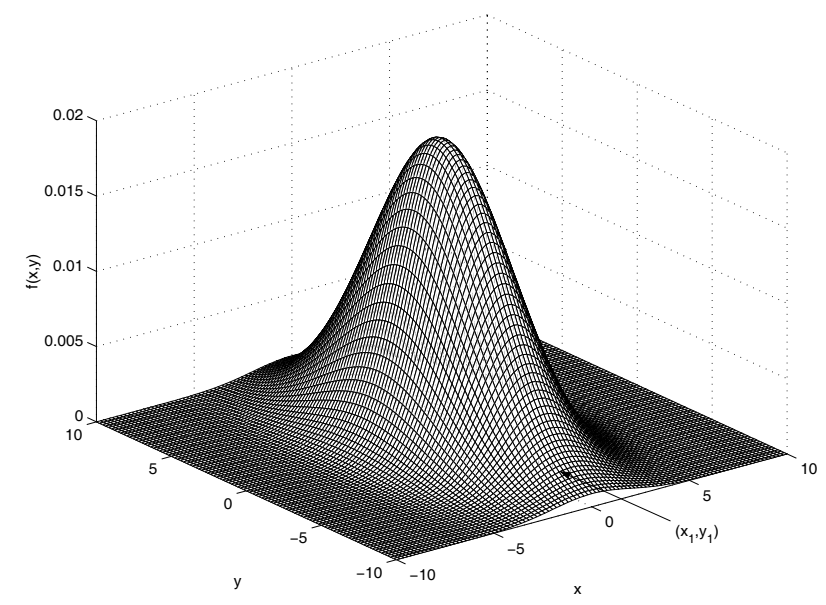

Fig. 3. $f(x, y)$ around $\mathcal{S}$. $(x, y) \in S_{p}$ defines a rangebearing bin centered on a point, $\left(x_{p}, y_{p}\right), p=1$, inside $\mathcal{S}$.

Let $H_{1}$ and $H_{0}$ represent respectively the events that an echo is or is not present in the range-bearing bin patch, $S_{p}$, centered on $\left(x_{p}, y_{p}\right) . x_{p}=R_{p} \cos \theta_{p}$ and $y_{p}=R_{p} \sin \theta_{p}$ where $\left(R_{p}, \theta_{p}\right)$ is the $p^{\text {th }}$ sample point in range-bearing space. The corresponding probabilities can be shown to be [6]

$$
\begin{aligned}
P\left(H_{1}\right) & =\iint_{S_{p}} f_{E}(x, y) d y d x \\
& \approx \frac{\left|S_{p}\right|}{2 \pi \alpha \beta r_{y}^{2}} e^{-\frac{r_{y p}^{2}}{2 \beta r_{y}^{2}}},, \\
P\left(H_{0}\right) & \approx 1-\frac{\left|S_{p}\right|}{2 \pi \alpha \beta r_{y}^{2}} e^{-\frac{r_{y p}^{2}}{2 \beta r_{y}^{2}}},
\end{aligned}
$$

where $\alpha=\frac{r_{x}}{r_{y}}$ and $r_{y p}^{2}=\frac{\left(x_{p}-\hat{x}_{n+1 \mid n}\right)^{2}}{\alpha^{2}}+\left(y_{p}-y_{n+1 \mid n}\right)^{2}$. $\alpha$ determines the orientation and elongation of $\mathcal{S}$, and $r_{y p}$ is the $y$ axis radius of an ellipse concentric with $\mathcal{S}$ which passes through $\left(x_{p}, y_{p}\right)$. All parameters are known and these expressions are easily evaluated at all range-bearing bins for any prediction region size.

\section{CONSTANT TOTAL PROBABILITY OF FALSE ALARM DETECTION}

The conventional detection approach is to threshold the magnitude squared output samples, $z\left(x_{p}, y_{p}\right)$, of a baseband quadrature digital matched filter receiver. $z$ is distributed central or noncentral $\chi^{2}$ with two degrees of freedom under $H_{0}$ or $H_{1}$ respectively. A threshold, $\tau$, is fixed so the probability of false alarm detection, $P_{F A}=\int_{\tau}^{\infty} f_{z}\left(z \mid H_{0}\right) d z$ is some small constant, e.g. $P_{F A}=10^{-7}$. Note that $P_{F A}$ is defined conditionally under $H_{0} . z>\tau$ indicates an echo detection.
We propose a new Bayesian framework for improved detection performance. The unconditional "total probability of false alarm" is defined as the joint probability that the threshold was crossed and no echo was present,

$$
\begin{aligned}
P_{T F A} & \triangleq P\left(z>\tau \cap H_{0}\right)=P\left(H_{0}\right) P_{F A}, \\
& =P_{\left(x_{p}, y_{p}\right)}\left(H_{0}\right) \int_{\tau\left(x_{p}, y_{p}\right)}^{\infty} f\left(z \mid H_{0}\right) d z,
\end{aligned}
$$

where the sampled spatial dependences of $P\left(H_{0}\right)$ and $\tau$ have been explicitly indicated.

We define the constant $P_{T F A}$ detector (CTFA) with the following steps:

1. Set $P_{T F A}=$ a small constant.

2. Solve (6) for the spatially varying $\tau\left(x_{p}, y_{p}\right)$.

3. Decide $H_{1}$ if $z\left(x_{p}, y_{p}\right)>\tau\left(x_{p}, y_{p}\right)$.

The effect of this approach is that the threshold is lowered in the predictions regions, $\mathcal{S}$ where based on prior track history it is known that echoes are more likely. This leads to more reliable detection of weak aircraft echoes. By proper design of the parameters of $f_{E}(x, y)$, the probability of detection, $P_{D}$, is increased while false alarms are kept low. Figure 4 illustrates how the variable threshold $\tau(x, y)$, might look along a 1-D slice through predictions regions for two tracks. Note that the lower quality track has a wider prediction region, leading to a more shallow, dispersed threshold depression. This corresponds to greater uncertainty about the next echo location, so less emphasis on detection is generated here. The decreased threshold regions should also not significantly increase $P_{F A}$ while it improves $P_{D}$. This is assured by carefully selecting larger values of $\beta$ for $f_{E}(x, y)$.

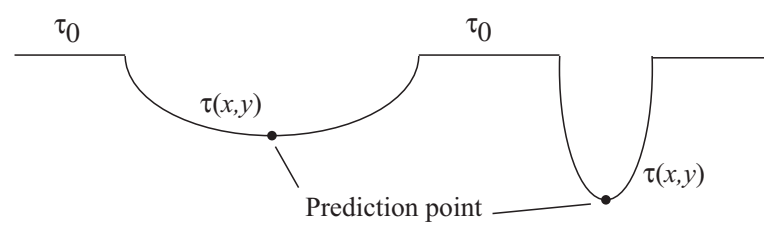

Fig. 4. Constant $P_{T F A}$ spatially varying detection threshold. $\tau(x, y)$ is at a local minimum corresponding to each prediction point.

\section{RESULTS}

To evaluate the detection performance improvement of the Bayesian CTFA algorithm under known, controlled conditions, a careful set of Monte Carlo simulation trials were 
completed. The simulation used realistic signal models (matching the ASR-3 RADAR, GBT receiver and aircraft motion), with the following simplifications. Only one echo track is simulated in each randomly generated trail. Pulse echo amplitude follows a Swirling distribution IV model for a square law receiver. Echo amplitudes are uncorrelated from pulse to pulse.

Figure 5 presents a set of ROC curves which demonstrate that the CTFA Bayesian algorithm has higher $P_{D}$ for a given $P_{F A}$ across all tested SNR levels. SNR is computed as an expectation at the matched filter ouptput.

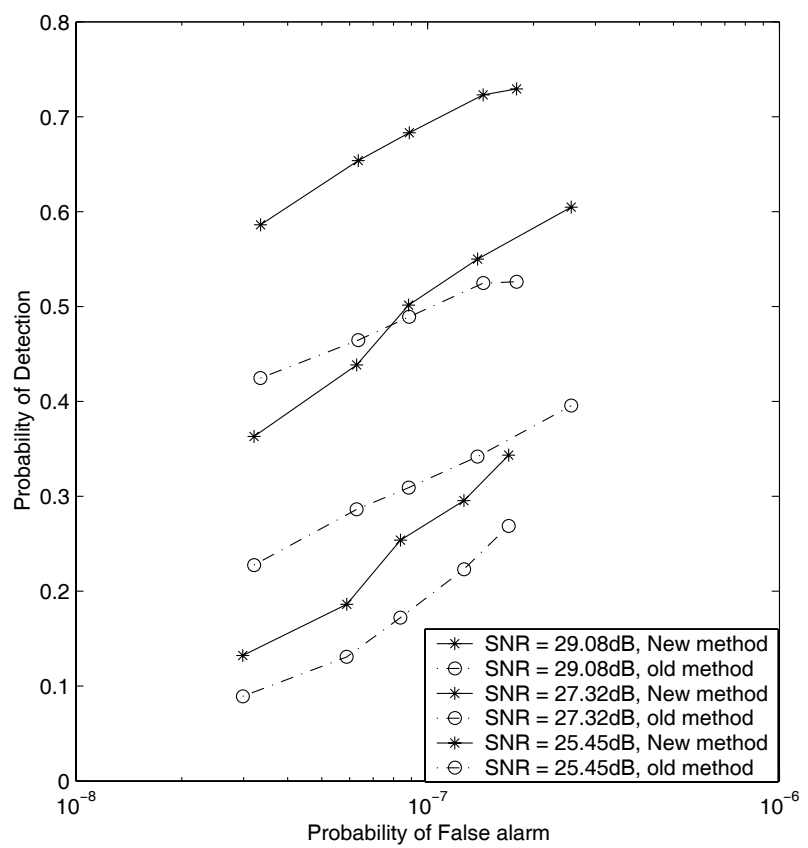

Fig. 5. $P_{F A}$ vs. $P_{D}$ for new Bayesian CTFA and "old" constant threshold algorithms. Top curve for each algorithm is $29.08 \mathrm{~dB}$ SNR, bottom is $25.45 \mathrm{~dB}$ SNR.

A set of real data recorded at the GBT for a $10 \mathrm{MHz}$ wide band around $1292 \mathrm{MHz}$ was used to test the echo detection algorithm, tracking, and blanking performance. By using the tracker information, the new Bayesian CTFA algorithm is able to detect some weaker echoes which were not detected by the conventional method. This was accomplished without increasing the false alarm rate. Figure 6 shows how each method performed in a data set with dense aircraft traffic. All detections were made by both algorithms, except those marked by rectangles, which only the Bayesian CTFA algorithm found. These detections are clearly in legitimate track positions.

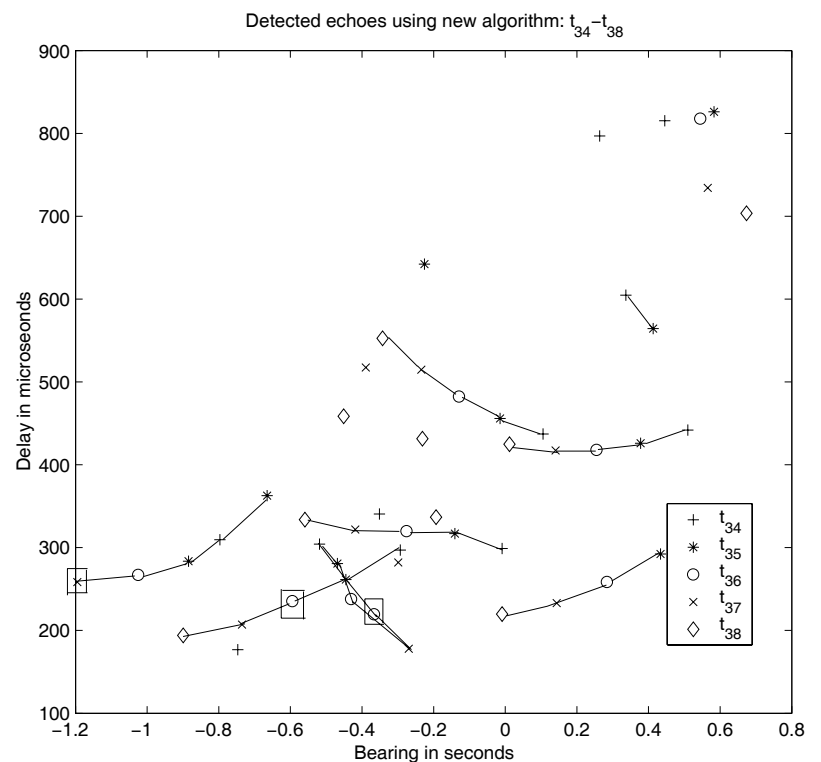

Fig. 6. Real data detection results for both algorithms. Note that 3 weaker pulses (inside the rectangles) are missed by the constant threshold algorithm, and picked up by CTFA.

\section{REFERENCES}

[1] Q. Zhang, Y. Zheng, S. Wilson, J. Fisher, and R. Bradley, "Combating pulsed radar interference in radio astronomy," The Astronomical Journal, vol. 126, pp. 1588-1594, 2003.

[2] S. Ellingson and G. Hampson, "Mitigation of radar interfernece in 1-band radio astronomy," The Astrophysical Journal Suplement Series, vol. 147, pp. 167-176, 2003.

[3] — - "A subspace-tracking approach to interference nulling for phased array-based radio telescopes," IEEE Trans. Antennas Propagat., vol. 50, no. 1, pp. 25-30, Jan. 2002.

[4] A. Leshem and A.-J. v.d. Veen, "Introduction to interference mitigation techniques in radio astronomy," in Perspectives in Radio Astronomy, Technologies for Large Antenna Arrays, A. Smolders and M. van Haarlem, Eds. Dwingeloo, The Netherlands: NFRA, 1999.

[5] K.V.Ramachandra, Kalman Filtering Techniques for Radar Tracking. Marcel Dekker, 2000.

[6] W. Dong, "Time blanking for gbt data with radar rfi," Master's thesis, Brigham Young University, 2004.

[7] W. Dong, B. Jeffs, and J. Fisher, "Real-time radar rfi blanking in radio astronomy using kalman tracking," to appear, Radio Science, 2005. 